\title{
Are House Prices in South Africa Really Non-Stationary? Evidence from SPSM-Based Panel KSS Test with a Fourier Function*
}

\author{
- Tsangyao Chang \\ (tychang@fcu.edu.tw) \\ (Department of Finance, Feng Chia University, Taichung, Taiwan) \\ - $\quad$ Tsung-pao $\mathrm{Wu}$ \\ (P9850573@fcu.edu.tw) \\ (Department of Finance, Feng Chia University, Taichung, Taiwan) \\ - Rangan Gupta \\ (Rangan.Gupta@up.ac.za)
}

(Department of Economics, University of Pretoria)

\begin{abstract}
This study applies the Sequential Panel Selection Method (SPSM) to investigate the time-series properties of provincial house prices for entire, large, medium, and small middle-segments of South Africa. Quarterly time-series data were collected from nine provinces in South Africa for different house-size categories over the period of 1978.Q1 to 2012.Q4. Whereas other panel-based unit root tests are joint tests of a unit root for all members of a panel and are incapable of determining the mix of integrated of order zero $(I(0))$ series and integrated of order one $(I(1))$ series in a panel setting, the SPSM proposed by Chortareas and Kapetanios (2009) can clearly identify how many and which series in the panel are stationary processes by classifying a whole panel into a group of stationary and non-stationary series. The empirical results from several panel-based, as well as standard pure time-series, unit root tests, indicate that house prices for the nine provinces studied here are either stationary or non-stationary. However, results from the SPSM using the
\end{abstract}

\footnotetext{
* We would like to thank two anonymous referees for many helpful comments. However, any remaining errors are solely ours.
} 
panel-version of the Kapetanios et al. (KSS, 2003) test with a Fourier function unequivocally indicate that house prices are stationary for the 9 provinces under study. Our test results have important economic and policy implications for South Africa.

Keywords: House Prices, Panel KSS Unit Root Test, Sequential Panel Selection Method, Non-Stationary JEL classification: C23, R21

\section{INTRODUCTION}

The recent world-wide financial crisis, emerging from the collapse of house prices, has necessitated careful investigation of the time-series properties of house prices. Whether house prices are characterized as stationary or non-stationary processes has important economic and policy implications. First, if house prices are characterized as an integrated of order one $(I(1))$ process, then the shocks affecting these series will have permanent effects, thus shifting the house price equilibrium from one level to another. The random walk (non-stationary) property also implies that the volatility of house prices can grow without bound in the long run, which has consequences for housing operation and regulation. In other words, if there are permanent effects from shocks to the house prices of a region, then this might need prolonged response from the government in terms of housing policy. On the other hand, if shocks to house prices are temporary, then policies should be ideally designed to have short-lived effects. Second, stationary properties of house prices are of great importance for economic modeling. Nelson and Plosser (1982) pointed out that whether data are modeled as a trend-stationary process or as a difference-stationary process, has important implications vis-à-vis modeling, testing and forecasting. For instance, when testing for causality between housing activities and macroeconomics, a precondition is that both variables need to be integrals of order one (characterized by a random walk). For policy-makers and finance professionals, Diebold and Kilian (2000) also propose that pre-testing for unit roots before applying forecasts yields superior forecasting performance, as opposed to the alternatives of always working with differenced series or always working with levels. Further, as is well-known, classical econometrics requires the use of stationary data to make valid inferences. However, if the variables in the system are cointegrated, then the first-differenced model needs to account for corrections to the long-run equilibrium to avoid model misspecification. All this requires pretesting of 
the data to ensure the appropriate order of integration of the variables under study.

Despite the important implications of stationarity or non-stationarity, no consensus has been reached in the empirical literature, which has primarily concentrated on the US and UK housing markets, and on house prices series. Meen (1999) and Peterson et al. (2002), using standard unit-root tests, find that the UK national house-price series follows a unit-root process. More recently, however, Cook and Vougas (2009) show that the use of a more sophisticated testing methodology can reverse findings derived using the conventional unit-root approach. Cook and Vougas (2009), using the smooth transition momentum-threshold autoregressive (ST-MTAR) test of Leybourne et al. (1998), confirm the stationarity characteristic of house-price changes but find that house prices exhibit structural change. Using quarterly data from 1975 to 1996 from the 50 US States, Muñoz (2003) finds unit roots in house-price changes, using the Dickey-Fuller Generalized Least Squares (DF-GLS) test (Elliott et al., 1996). Meen (2002) compares the time-series behavior of house prices in the US and UK. Using quarterly data from 1976 to 1999 for the US and from 1969 to 1999 for the UK, Meen (2002) conducts both Augmented Dickey-Fuller (ADF) and Phillips-Perron (PP) unit-root tests on the level of house prices and finds that in both countries house prices follow a first-differenced stationary process. That is, house prices are integrated of order one. Canarella et al. (2012) employed linear and nonlinear unit-root tests to investigate the price dynamics of the house price indices included in the S\&P/Case-Shiller Composite10 index. In general, the findings lack uniformity and tended to depend upon the assumptions imposed by the testing procedures. The tests that assume structural stability and linear adjustment failed to provide evidence in favor of stationarity in the price dynamics of all series. Conversely, the nonlinear test of Kapetanios et al. (2003) provided evidence that the price dynamics of Los Angeles and San Francisco follow a nonlinear stationary process. The Lumsdaine and Papell (1997) and Lee and Strazicich (2003) tests indicated that significant structural breaks exist in all series. However, whereas the Lumsdaine-Papell test found evidence of trend-stationarity with breaks only in the price dynamics of Las Vegas, the Lee-Strazicich test found the price dynamics of Miami and San Diego also exhibit broken-trend stationarity.

We are not surprised about the diversity of these test results as divergence tends to result from inaccurate methodologies applied to heterogeneity housing development in different regions and sectors. 
Series of house prices actually reflect the evolving path of the housing-construction industry which adapts to different regions and different stages of development. Thus, it is always meaningful to test house prices series at the regional level. Against this backdrop, the objective of this paper is to analyze whether provincial-level house prices in South Africa are stationary or non-stationary using not only standard time-series-based unit root tests, but also panel unit root tests to allow for cross-sectional dependence across the regions. Note that while the provincial-level data is categorized on all sizes taken together, it is further categorized based on house sizes, namely, large, medium and small, to allow for the role of heterogeneity across different income categories involved in the housing market. The decision to use South Africa as a case study has several motivations: first, we are familiar with the housing market and availability of data for over long spans and across regions, coupled with the general lack of any study on unit root properties of house prices dealing with an emerging economy; second, to the best of our knowledge, there exist no studies on South Africa that deal with unit root properties of house prices at the provincial level. Primarily, while analyzing univariate characteristics of house prices, researchers in South Africa (Burger and van Rensburg, 2008; Das et al., 2010; Balcilar et al., 2013) have concentrated on the convergence of and the ripple effect of house prices across major metropolitan areas of South Africa using a wide range of unit root testing procedures, with the appropriate metric being relative house prices rather than the absolute nominal house price. Other studies (Simo-Kengne et al., 2012, 2013) have looked at unit root properties of real house prices across provinces, using standard panel unit root tests, and have analyzed the spillover effect of the housing sector onto the real economy. ${ }^{1}$ Third, and perhaps most importantly, given that South Africa is an inflation targeting country, ${ }^{2}$ the stationarity or non-stationarity property of house prices is of paramount importance, since it is likely to impact on the behaviour of the aggregate inflation of the economy. This has been pointed out by Gupta and Hartley's (forthcoming) finding that house prices lead inflation (and real economic activity) in South Africa, which is not surprising given that housing accounts for $29.4 \%$ of household assets and

1 Gil-Alana et al. (forthcoming) is the only other study to have looked at persistence properties of nominal house prices of South Africa, based on ARFIMA models. However, this study concentrates on the different national segments of the housing market, namely, luxury, middle (entire, large, medium and small) and affordable. See Section 3 on the discussion of housing data for further details regarding this classification.

2 Since the announcement made by the minister of Finance in the February of 2000, the sole objective of the South African Reserve Bank (SARB) has been to achieve and maintain price stability. More specifically, the SARB has now adopted an explicit inflation targeting regime, whereby it aims to keep the Consumer Price Index (CPI) inflation within the target band of 3 percent to 6 percent, using discretionary changes in the Repurchase (Repo) rate as its main policy instrument. 
$21.68 \%$ of total wealth (Gil-Alana et al., forthcoming). Increase (decrease) in house prices following an increase (decrease) in housing demand, would lead to an increase (decrease) in residential investment, which in turn, would cause aggregate demand to increase (decrease), resulting in inflationary (deflationary) pressures. Hence, it is important to understand whether shocks to house price would have permanent or temporary effects on house prices, which in turn, will determine whether the effect on inflation could possibly a short-lived or prolonged one, so that monetary authority can react accordingly. In this regard, also note that our panel-based unit root testing approach enables us to not only account for cross-sectional dependence due to spatial spillovers (thus allowing us to treat the housing market at a regional rather than a national level), but also to exactly identify which of the regions in the panel are characterized by stationary or non-stationary house prices. This information is of tremendous importance to policy makers, since it allows them to know exactly how a house price shock in one region is likely to affect the mean-reversion or explosive behaviour of domestic inflation. However, the above line of reasoning to some extent is conjectural, since our paper only concentrates on house prices, and does not look at the channel through which house prices can possibly affect inflation. But, recent papers by Simo-Kengne et al., (2012, 2013, forthcoming a, b), based on both regional and aggregate analysis, have indicated of significant impact of South African house prices on inflation.

In the same vein as Balcilar et al. (2011), who highlight the existence of nonlinear behaviour in South African house prices, we also account for the same in our unit root testing procedure. It must be realized that the behaviour of the housing market is not the same across phases of expansion and contraction that characterize the real estate sector (Kim and Bhattacharya, 2009). Seslen (2004) argued that households exhibit forward looking behaviour and have a higher probability of trading up during the upswing when equity constraints are less binding. However, the same is not true during the downswing of the housing market cycle, since when house prices are on the decline, households are less likely to trade; thus there is some downward rigidity of house prices. The aversion to loss during the downswing is more than likely to reduce the mobility of households as far as trading is concerned. Further, as pointed out by Muellbauer and Murphy (1997), the presence of lumpy transaction costs in the housing market can also cause non-linearity. Given these issues, it is important and makes sense to incorporate non-linearity in the data generating 
process of housing prices. Further note that, by using provincial-level house prices, as well as, different house sizes, we consider the existence of heterogeneity of economic development, socio-cultural factors, and other national circumstances across the regions and income categories. Nonlinearities are likely to emerge with the development of housing markets being different for across income levels and provinces.

Canarella et al. (2012) write that the mixed international evidence that exists on unit root properties of house prices could be a result of possible shortcomings in the econometric models of past studies. It has been widely reported that conventional unit root tests, on which most of the international literature is based, not only fail to consider information across regions (an issue of tremendous importance when working with housing markets, which in turn, are likely to be more regional in nature than to have a uniform national structure); thus conventional unit root tests have less efficient estimations, and lower power than the near-unit-root but stationary alternatives. In order to increase the power of tests for a unit root, many researchers have employed panel data (Taylor and Sarno, 1998; Maddala and Wu, 1999; Levin et al., 2002; Im et al., 2003). However, panel tests are not informative in terms of the number of series that are stationary processes when the null hypothesis is rejected. The reason is simple: they are not joint tests of the null hypothesis. In this regard, Breuer et al. (2001) claimed that, by analogy to a simple regression, when an $F$-statistic rejects the null that a vector of coefficients is equal to zero, it is not necessarily true that each coefficient is nonzero. Likewise, when the unit-root null hypothesis is rejected, it may very well not be justified to assume that all series in the panel are stationary.

Panel-based unit root tests that are joint tests of a unit root for all members of a panel are incapable of determining the mix of $\mathrm{I}(0)$ and $\mathrm{I}(1)$ series in a panel setting. By contrast, the Sequential Panel Selection Method (SPSM), proposed by Chortareas and Kapetanios (2009), classifies a whole panel into a group of stationary series and a group of non-stationary series. In doing so, it clearly identifies how many and which series in the panel are stationary processes.

Another possible reason for the mixed international evidence could be the due to the fact that the standard unit root tests do not often account for structural breaks and/or nonlinearities. As highlighted by Canarella et al. (2012), conventional unit root tests, which assume structural stability and linear adjustment, can interpret departures from linearity and structural instabilities as permanent stochastic disturbances, i.e., sway the 
analysis toward the unit root hypothesis. The general method to account for breaks is to approximate them using dummy variables. However, this approach has several undesirable consequences: first, one has to know the exact number and location of the breaks. These are not usually known and therefore need to be estimated. This in turn introduces an undesirable pre-selection bias (see Maddala and Kim, 1998). Second, currently available time-series based unit root tests account only for one to two breaks. Third, the use of dummies suggests sharp and sudden changes in the trend or level. However, for low frequency data, such as quarterly house price data, it is more likely that structural changes in the housing sector take the form of large swings which cannot be captured well using only dummies. So breaks in house prices should therefore be approximated as smooth and gradual processes (see Leybourne et al., 1998; Balcilar et al., 2011; and Footnote 5 of this paper for further evidence along these lines). These arguments motivate the use of a recently developed set of unit root and stationary tests that avoid this problem. Both Becker et al. (2004, 2006) and Enders and Lee $(2004,2009)$ develop tests which model any structural break of an unknown form as a smooth process via means of flexible Fourier transforms. Several authors, including Gallant (1981), Becker et al. (2004), Enders and Lee (2004, 2009), and Pascalau (2010), show that a Fourier approximation can often capture the behavior of an unknown function even if the function itself is not periodic. The authors argue that their testing framework requires only the specification of the proper frequency in the estimating equations. By reducing the number of estimated parameters, they ensure the tests have good size and power irrespective of the time or shape of the break. Additionally, the existence of structural changes in house prices might imply broken deterministic time trends and might result in a nonlinear pattern (Bierens, 1997). It should, therefore, not be unexpected that these shortcomings have seriously called into question many of the earlier findings based on unit roots in house prices.

In light of this, our paper tests the stationarity of regional house prices across different size-categories in South Africa using the Seemingly Unrelated Regression version of Kapetanios et al.'s (2003, SURKSS) unit root test based on the SPSM, with a Fourier function. We believe that this is the first study in the unit root testing literature of house prices that uses and combines SURKSS based on the SPSMS with a Fourier transform to test the stationarity hypothesis, using South Africa as a case study. The paper is organized as follows: Section 2 outlines the methodology; Section 3 presents the data, Section 4 discusses the empirical 
findings. Section 5 discusses economic and policy implications, Section 6 provides concise conclusions.

\section{METHODOLOGY}

Studies have found that many macroeconomic and financial time series not only contain unit roots but also exhibit nonlinearities. Conventional unit root tests, such as the ADF unit root test, have low power in detecting the mean-reverting tendency of the series. For this reason, stationary tests in a nonlinear framework must be applied. Here, we use the nonlinear stationary test advanced by Kapetanios et al. (2003) (KSS).

In line with Kapetanios et al. (2003), the KSS test is based on detecting the presence of non-stationarity against a nonlinear but globally stationary exponential smooth transition autoregressive (ESTAR) process. The main idea is that time series data may revert to the mean only when they are sufficiently far away from it. When they are close to their mean, they may behave as non-stationary processes. Accordingly, the model is given by

$$
\Delta H P_{t}=\gamma H P_{t-1}\left\{1-\exp \left(-\theta H P_{t-1}^{2}\right)\right\}+v_{t}
$$

where $H P_{t}$ is the house price data series of interest, $v_{t}$ is an i.i.d. error with zero mean and constant variance, and $\theta \geq 0$ is the transition parameter of the ESTAR model and governs the speed of transition. Under the null hypothesis $H P_{t}$ follows a linear unit root process, but under the alternative hypothesis, $H P_{t}$ follows a nonlinear stationary ESTAR process. One shortcoming of this framework is that the parameter $\gamma$ is not identified under the null hypothesis. Kapetanios et al. (2003) used a first-order Taylor series approximation for $\left\{1-\exp \left(-\theta I P_{t-1}^{2}\right)\right\}$ under the null hypothesis $\theta=0$ and then approximated equation (1) by using the following auxiliary regression:

$$
\Delta H P_{t}=\xi+\beta H P_{t-1}^{3}+\sum_{i=1}^{k} b_{i} \Delta H P_{t-i}+v_{t}, t=1,2, \ldots, T
$$

In this framework the null hypothesis and alternative hypotheses are expressed as $\beta=0$ (non-stationarity) against $\beta<0$ (non-linear ESTAR stationarity). Ucar and Omay (2009) (referred to as the UO test hereafter) expanded a nonlinear panel data unit root test based on regression (2). The regression is:

$$
\left.\Delta H P_{i, t}=\gamma{ }_{i} H P_{, i}\left\{1-\exp \theta \quad H_{-,}^{2} P_{i l t}+\right)\right\}
$$


UO also applied a first-order Taylor series approximation to the Panel ESTAR (3) model around $\theta_{i}=0$ for all $i$, and obtained the auxiliary regression:

$$
\Delta H P_{i, t}=\xi_{i}+\beta_{i} H P_{i, t-1}^{3}+\sum_{j=1}^{k} \theta_{i, j} \Delta H P_{i, t-j}+v_{i, t}
$$

where $\beta_{i}=\theta_{i} \gamma_{i}$ and the hypotheses established for unit root testing based on regression (4) are as follows:

$$
\begin{aligned}
& H_{0}: \beta_{i}=0, \text { for all } i, \text { (linear nonstationarity) } \\
& H_{1}: \beta_{i}<0, \text { for some } i, \text { (nonlinear stationarity) }
\end{aligned}
$$

Furthermore, the system of KSS equations with a Fourier function that we estimate here is:

$$
\Delta H P_{i, t}=\xi_{i}+\delta_{i} H P_{i, t-1}^{3}+\sum_{j=1}^{k 1} \theta_{i, j} \Delta H P_{i, t-j}+a_{i, 1} \sin \left(\frac{2 \pi k t}{T}\right)+b_{i, 1} \cos \left(\frac{2 \pi k t}{T}\right)+\varepsilon_{i, t}
$$

where $t=1,2, \ldots ., T$. The rationale for selecting $[\sin (2 \pi k t / T), \cos (2 \pi k t / T)]$ is based on the fact that a Fourier expression is capable of approximating absolutely integrable functions to any desired degree of accuracy. $k$ represents the frequency selected for the approximation, and $\left[a_{i}, b_{j}\right]^{\prime}$ measures the amplitude and displacement of the frequency component. It also follows that at least one frequency component must be present if there is a structural break. Gallant (1981), Becker et al. (2004), Enders and Lee (2012), and Pascalau (2010), showed that a Fourier approximation can often capture the behavior of an unknown function even if the function itself is not periodic. As there is no apriori knowledge concerning the shape of the breaks in the data, a grid-search is first performed to find the best frequency. Following Enders and Lee (2012), we first set a maximum value of $k=5$, and then run the test for: $k=1,2,3,4$ and 5, to get an optimum value of $k=k^{*}$, corresponding to which the sum of squared residuals is minimized. Then we use this optimum $k^{*}$ to obtain our panel tests.3 Understandably, $k^{*}$ varies across the cross-sectional units (as well as the various sub-section of the housing market, details of which has been discussed below).

Since we use the SURKSS tests with a Fourier function in conjunction with the SPSM, we now discuss the SPSM. The SPSM proposed by Chortareas and Kapetanios (2009) is based on the following steps:

(1) The Panel KSS test with/without the Fourier function is first conducted on the log-values of all the house prices $H P_{t}$ in the panel for a specific size-category, as well as for all the sizes taken together. If the null of unit-root cannot be rejected, the procedure is stopped, and all the series in the panel are concluded to be non-stationary. If the null is rejected, we go to Step 2;

(2) We remove the series with the minimum KSS statistic since it has been identified as stationary;

3 A MATLAB do-loop code was developed for this purpose of the test, and is available upon request from the authors. 
(3) We return to Step 1 for the remaining series, or stop the procedure if all the series have been removed from the panel.

The final result is a separation of the whole panel into a set of mean-reverting series and a set of non-stationary series.

\section{DATA}

We use seasonally adjusted quarterly house price indexes, with data taken from Amalgamated Bank of South Africa (ABSA). ABSA categorizes housing into three price segments, namely luxury (ZAR 3.5 million ZAR 12.8million), middle (ZAR 480,000 - ZAR 3.5 million) and affordable (below ZAR 480,000 and area between 40 square metres - 79 square metres). The middle-segment is further categorized into three more segments based on sizes, namely large-middle (221 square metres - 400 square metres), medium-middle (141 square metres - 220 square metres) and small-middle (80 square meters - 140 square meters). Provincial level data is, however, only available for the entire (all) middle-segment and its various categories based on size. To ensure a balanced panel, our data starts from 1978.Q1 and ends in 2012.Q4. The raw datasets for house prices indicate that Western Cape and Northern Cape, respectively, have the highest and lowest mean house prices (for all, large, medium, small middle-segments), as shown in Table 1. The Jarque-Bera test results indicate that the datasets for all different house prices collected for 9 provinces are approximately non-normal. All unit root tests are conducted on the natural-logarithm of house prices.

\section{Empirical Results}

\subsection{Unit Root Tests}

Several univariate time series unit root tests are first employed to examine the null of a unit root for house prices for the 9 provinces of South Africa. Then, both first-generation and second-generation panel unit root tests are employed. Tables 2-5 report the results of the three univariate unit root tests - the Augmented Dickey and Fuller (1981, ADF), the Phillips and Perron (1988, PP), and the Kwiatkowski et al. (1992, KPSS) tests. All tests lead us to conclude that house prices (for Large, Medium, Small, and All-segment) of the 9 provinces in South Africa contain unit roots. This result is consistent with the existing literature and may be due to the low power of these three univariate unit root tests when the house prices are highly persistent. 
Another reason for finding (possible spurious) unit roots could be, as have recently been argued, that house prices are likely to be non-linear due to the existence of transaction costs and hence the power of these three tests might be poor in such situations (Wu and Lee, 2009). Furthermore, we know that univariate unit root tests might have low power when they are applied to a finite sample. In this situation, panel-based unit tests are found to be of great help, provided that they allow for an increase in the power of the analysis by allowing the cross-sectional and temporal dimensions to be combined.

\subsection{First and Second Generation Panel Unit Root Tests}

Tables 6 and 7 report the results for the first-generation and second-generation panel-based unit root tests for house prices of the large, medium, small and the entire middle-segments, respectively. By looking at Table 6 shows that the first-generation panel-based unit root test of the LLC (Levin et al., 2002) yield similar results indicating that house prices for large, medium, small and the entire middle-segments, respectively, in all 9 provinces of South Africa are stationary. However, the results from the IPS (Im et al., 2003), Maddala and Wu (1999) and Hadri (2000) tests indicate that house prices (for large, medium, small and the entire middle-segments) are non-stationary. Table 7 shows that four second-generation panel-based unit root tests yield different results. Based on Table 7, we find that both Bai and Ng (2004), Choi (2002) and Chang (2002) IV test results indicate that house prices for the large, medium, small and the entire middle-segments, are non-stationary. However, results from both Moon and Perron (2004) and Pesaran (2007) tests indicate that house prices for the large, medium, small and the entire middle-segments, are stationary.

As stated earlier, both the first and second-generation panel-based unit root tests are joint tests of a unit root for all members of a panel, and hence, are incapable of determining the mix of $I(0)$ and $I(1)$ series in a panel setting. To identify how many areas, and which areas in the panel support the non-stationary process, we proceed to the SPSM procedure combined with the Panel KSS unit root test.

\subsection{Panel KSS Test using Sequential Panel Selection Method (SPSM)}

Table 8 reports the results from the Panel KSS unit root test with a Fourier function on house prices for large, medium, small and the entire middle-segments. Table 8 reports a sequence of the Panel KSS statistics with their bootstrap $p$-values ${ }^{4}$ on a reducing panel, the individual minimum KSS statistic, and the stationary

4 The asymptotic $p$-values are computed by means of Bootstrap simulations using 10,000 replications. 
series identified by this procedure for each value. If we look at the large middle-segment in Panel A of Table 8 , we find that the null hypothesis of unit root in house price is rejected, when the Panel KSS unit root test is first applied to the whole panel, producing a value of -5.0204 with a very small $p$-value of 0.000 . After implementing the SPSM procedure, we find North West is stationary with the minimum KSS value of -3.4872 among the panel. North West is then removed from the panel and the Panel KSS unit root test is implemented again to the remaining set of series. After that, we find that the Panel KSS unit root test still rejected the unit root null with a value of -4.9818 (p-value of 0.000 ), and KwaZulu-Natal is found to be stationary with the minimum KSS value of -3.1233 in the panel this time. KwaZulu-Natal is then removed from the panel and the Panel KSS unit root test is implemented again for the remaining set of series. The procedure is continued until the Panel KSS unit root test fails to reject the null hypothesis of unit root at the $10 \%$ significance level, and finally we find that this procedure stops at the ninth sequence, when the house prices for all the nine provinces have been removed from the panel. Thus, the SPSM procedure using the Panel KSS unit root test with a Fourier function provides strong evidence favoring the mean-reverting in house prices for all the nine provinces of the large middle-segment. Panels B, C, and D of Table 8 report the test results for the hours prices of medium, small, and entire middle-segments, respectively, and also yield similar results to those of the large middle-segment, indicating house prices in all the sections of the middle-segment across the 9 provinces in South Africa are characterized by a mean-reverting processes, i.e., they are stationary.

As a benchmark, we also report the results of Panel KSS unit root test without a Fourier function on house prices of the large, medium, small and the entire middle-segments. Table 9 reports a sequence of the Panel KSS statistics with their bootstrap $p$-values ${ }^{5}$ on a reducing panel, the individual minimum KSS statistic, and the stationary series identified by this procedure for each time. If we look at Panels A, B, C, and $\mathrm{D}$ of Table 9, we can find that the null hypothesis of unit root in house prices cannot be rejected for all the middle-segment sections across the nine provinces. The results from Tables 8 and 9 point to the importance of proper modeling of structural breaks and nonlinearities in house prices for South Africa.

Figures 1, 2, 3, 4 display the time paths of house prices for large, medium, small, and entire

5 The asymptotic $p$-values are computed by means of Bootstrap simulations using 10,000 replications. 

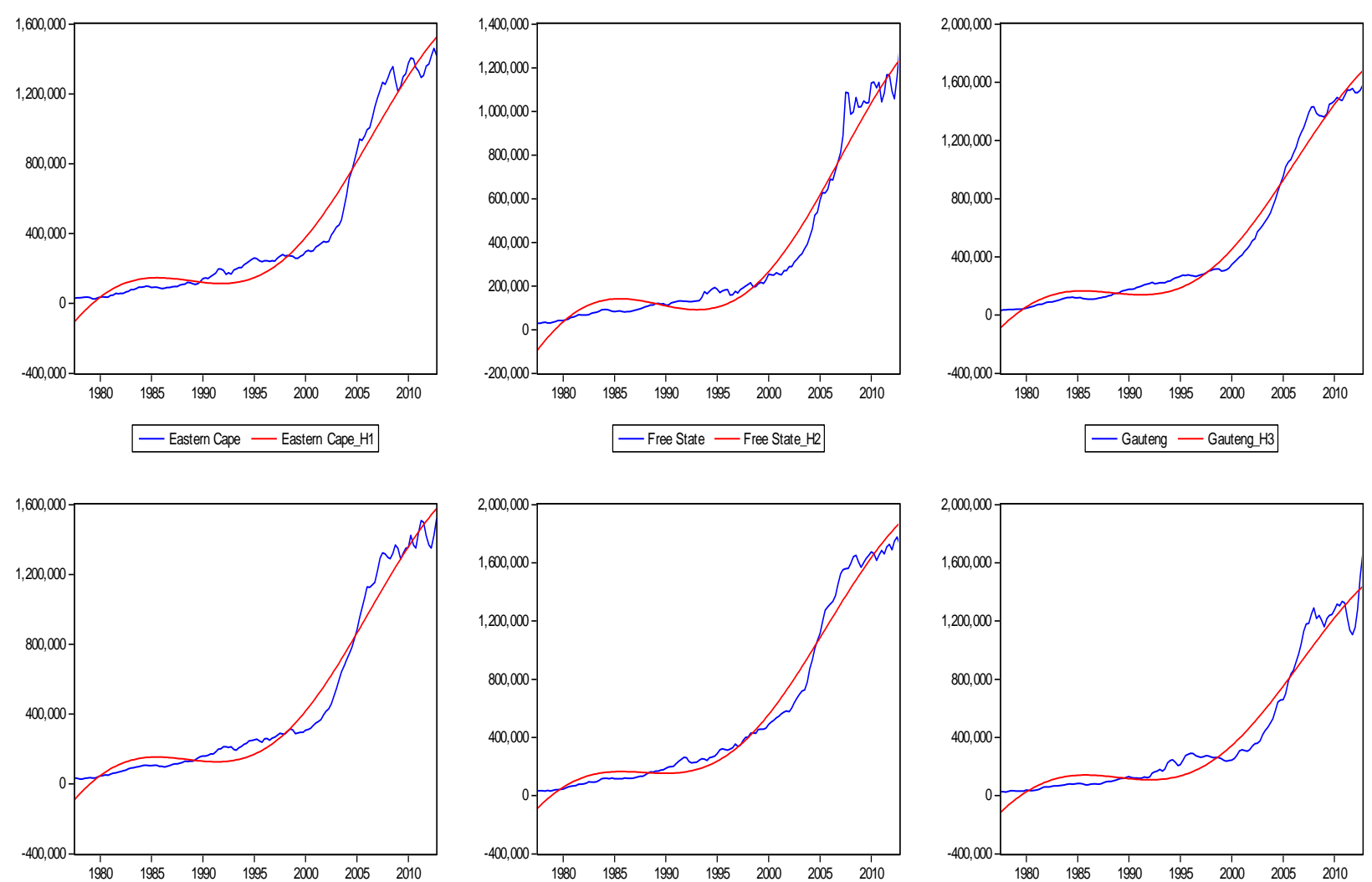

- KwaZulu-Natal —KwaZulu-Natal_H4
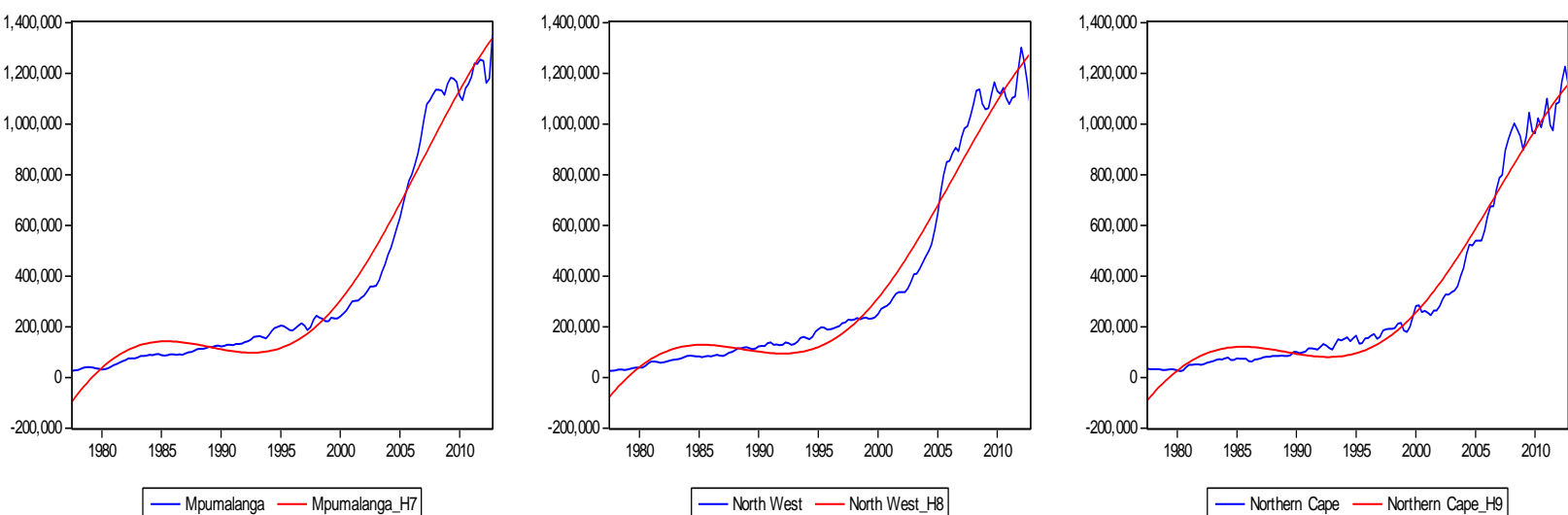

- Mpumalanga —Mpumalanga_H7

- North West

— Northern Cape — Northern Cape_H9

Figure 1. Plots of large middle-segment house prices and fitted nonlinearity 


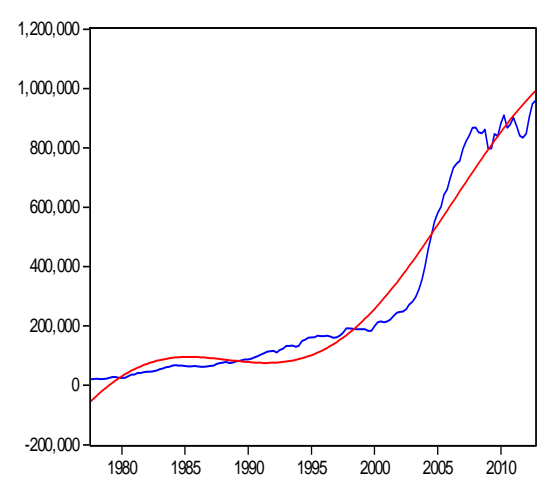

—Eastern Cape —Eastern Cape_H1

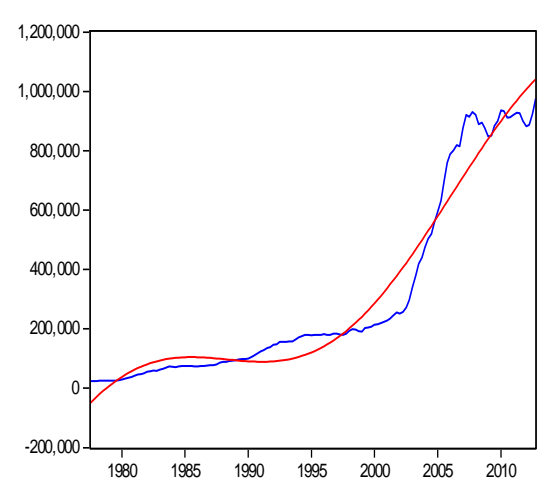

- KwaZulu-Natal —KwaZulu-Natal_H4

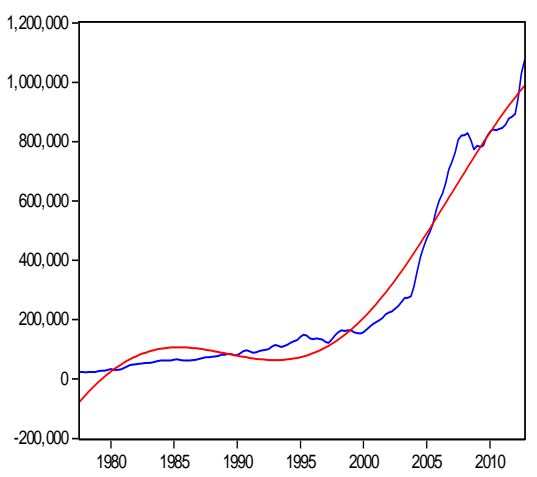

- Mpumalanga - Mpumalanga_ $\mathrm{H7}$

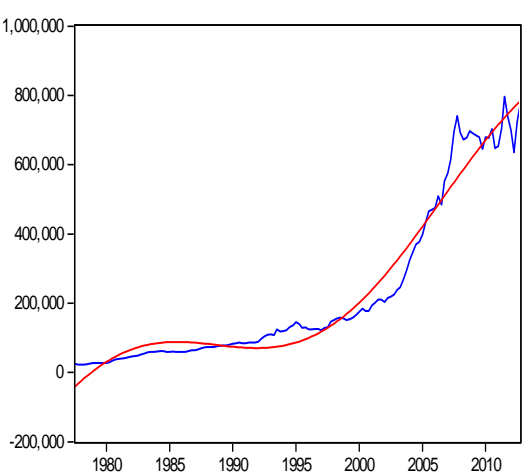

一 Free State —Free State_H2

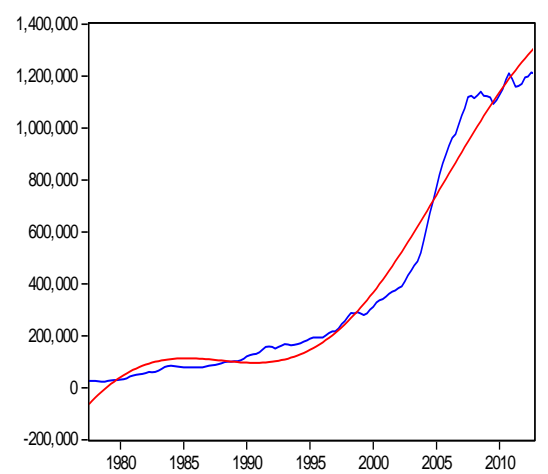

- Western Cape —Western Cape_H5

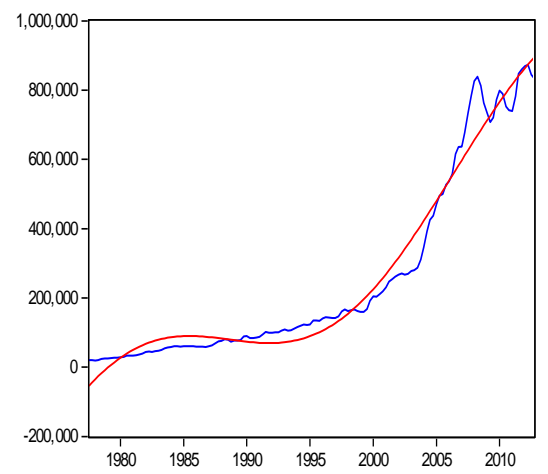

— North West — North West_H8

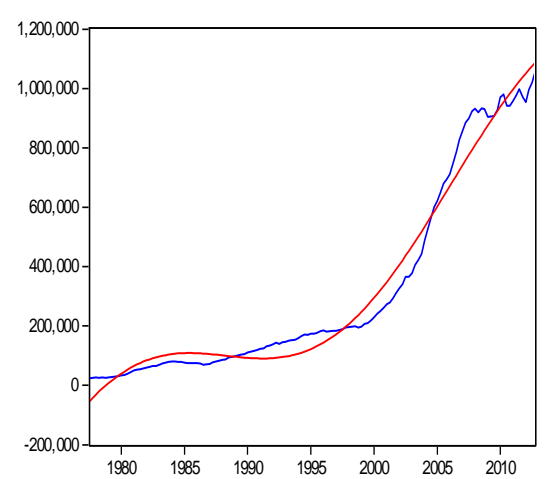

- Gauteng - Gauteng_H3

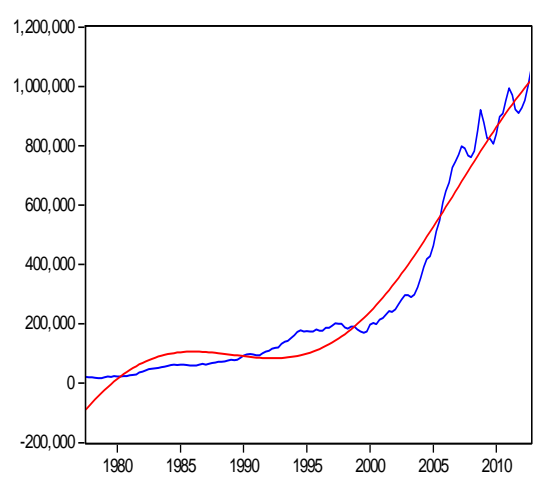

— Limpopo — Limpopo_H6

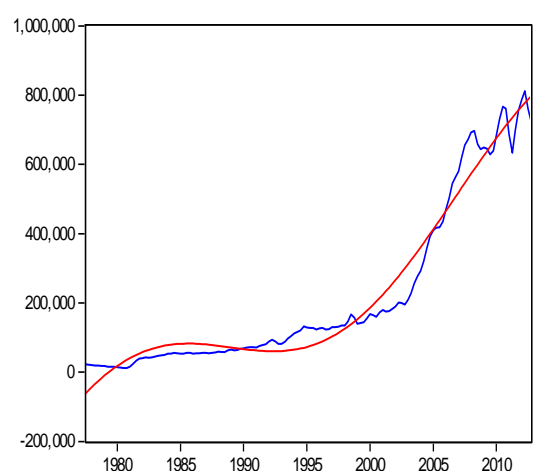

— Northern Cape — Northern Cape_H9

Figure 2. Plots of medium middle-segment house prices and fitted nonlinearity 

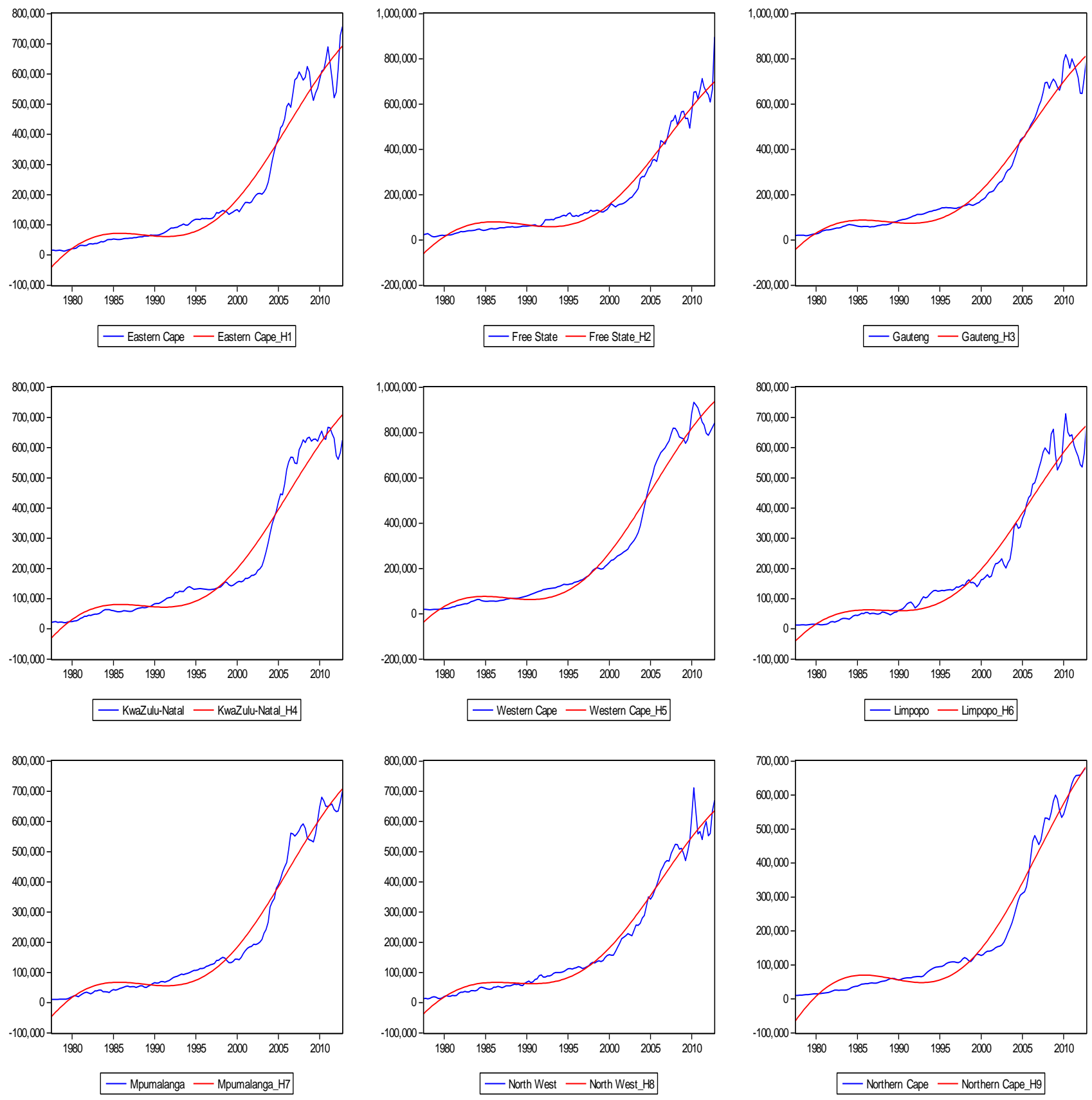

Figure 3. Plots of small middle-segment house prices and fitted nonlinearity 

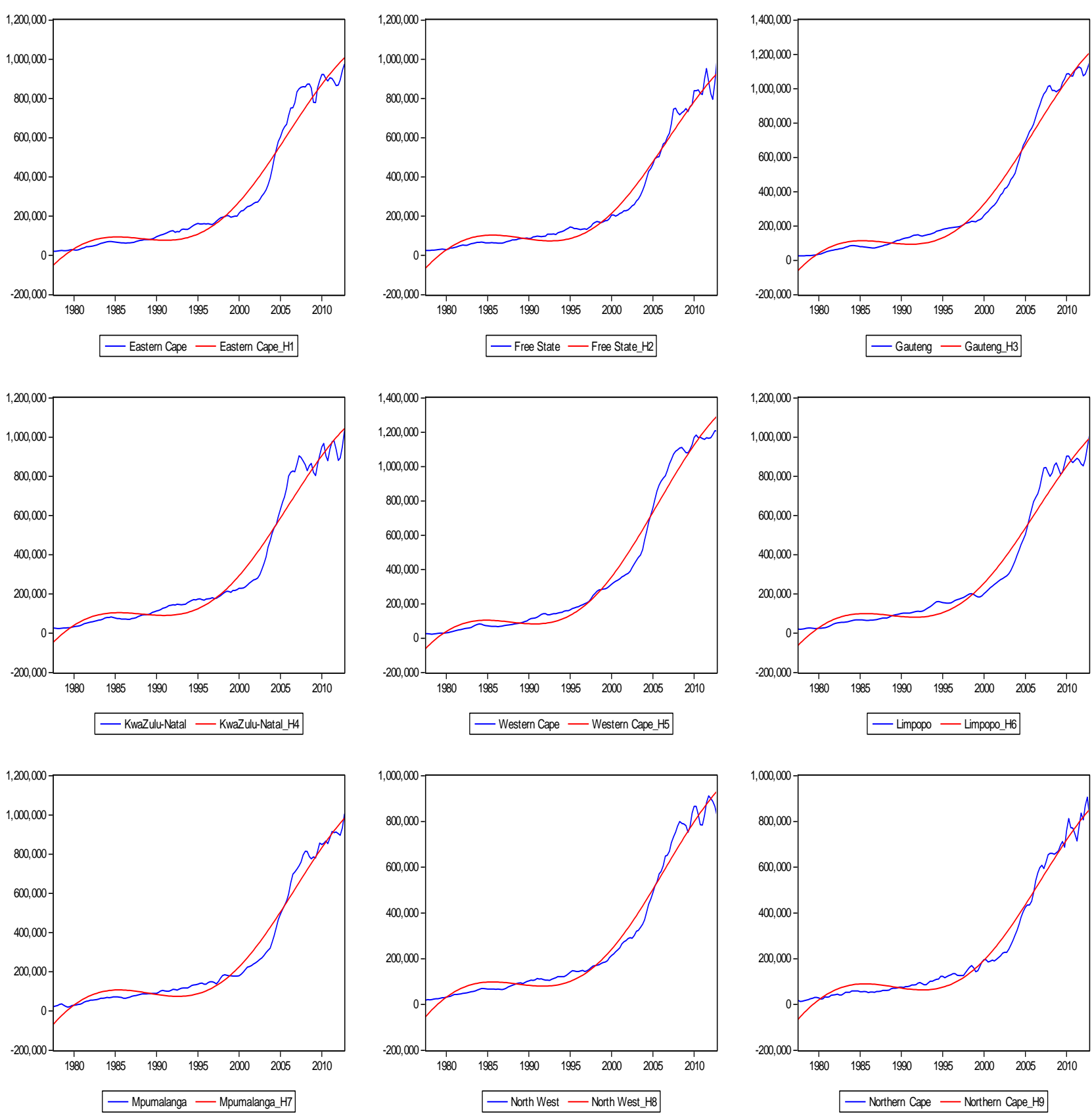

Figure 4. Plots of entire middle-segment house prices and fitted nonlinearity

middle-segments, respectively. We can clearly observe structural shifts in the trend of the data after the 2000s. Accordingly, it appears sensible to allow for structural breaks in testing for a unit root (and/or stationarity). The estimated time paths of the time-varying intercepts are also shown in Figures 1, 2, 3, and 4. As we know that the actual nature of break(s) is generally unknown, and there is no specific guide as to where and how many breaks should be used in testing for a unit root or stationarity, using an incorrect specification for the form and number of breaks can be as problematic as ignoring the breaks altogether. A 
further examination of the figures indicates that all the Fourier approximations seem reasonable and support the notion of long swings in house prices of the 9 provinces for the large, medium, small, and entire middle-segments, respectively. ${ }^{6}$

\section{Economic and Policy Implications}

Several economic and policy implications emerge from our study: First, if the data were erroneously treated as non-stationary and causality tests for house prices and other macroeconomic variables were applied to the first difference, then a spurious causality would be seen, because the non-stationary behavior would not be transmitted to other macroeconomic variables through housing activities if the latter followed a mean-reverting process. As Nelson and Plosser (1982) pointed out, whether a data series is modeled as a trend-stationary or a difference-stationary process has important implications for modeling, testing, and forecasting. Second, in the present study, overwhelming evidence in favor of the $\mathrm{I}(0)$ stationary hypothesis is found, implying that for most provinces in South Africa, shocks to house prices are not permanent. This result suggests that following a major structural change, say, from the global financial markets, house prices will return to their original equilibrium over a period of time. If shocks to house prices are temporary, there are short-lived effects for housing market-related policies, and hence, the government can afford to wait before intervening in the process of reversion. On the other hand, stationarity of house prices in most provinces of South Africa also indicates that the prospects of real estate development in South Africa are, in general, stable and positive, since there does not seem to evidence of housing market bubbles - result also confirmed by Chang et al., (forthcoming). This is of interest to various parties, including foreign and domestic investors and local governments. Furthermore, our empirical findings also suggest that allowing

6 We also tested for the mean-reversion behaviour in the house prices using the SURADF test proposed by Breuer et al. (2001). The test indicated provided very mild evidence of stationarity (3 cases at most) at the five percent level of significance. The number went up to 5 in one instance (medium middle-segment) when we considered a significance level of 10 percent. The weak performance of the SURADF test in detecting mean-reversion is not surprising given the evidence provided by Wu and Lee (2009) of superior power of the SURKSS test relative to the SURADF test. In addition to the SURADF test, we also tested for the stationarity of house prices using the panel unit root tests with multiple structural breaks (using a maximum of 5 breaks in our case), as proposed by Carrion-i-Silvestre et al, (2005). Most of the series displayed 3 breaks (early 1990s when house prices in South Africa started booming, early 2000 when inflation targeting was adopted and in between late 2006 and mid-2007 during the financial crisis when the housing markets started to slow down around the world), but with virtually no evidence of stationarity, especially when cross-sectional dependence was considered, as suggested by the test proposed by Pesaran (2004). The details of all these tests are available upon request from the authors. More importantly though, these results highlight the importance of using the SURKSS test over the SURADF test and the panel unit root test with multiple structural breaks, in terms of not only using a more powerful test, but also modeling structural changes in the data using a smooth process to accommodate the fact that transition from one regime to another is not abrupt, but is a smooth process. The need to model middle-segment house prices using a smooth transition is in line with the evidence provided by Balcilar et al. (2011). 
for nonlinearities and structural breaks results in more rejection of the null hypothesis unit root, which is consistent with the mean-reverting process of house prices. The results point to the importance of proper modeling of structural breaks and nonlinearities in house prices series for South Africa.

\section{Conclusions}

The main purpose of this article is to adopt the newly developed and refined nonlinear panel unit-root test (KSS test with a Fourier function) by UO based on a SPSM to test for the stationarity of house prices in nine provinces of South Africa, with the house prices being further disaggregated based on housing size to account for the impact of income levels on the results. The investigation is carried out for the period of 1978:Q1-2012:Q4. The development of real estate markets across provinces justifies the application of nonlinear models, due to the heterogeneity of economic development and socio-cultural factors, as well as the imperfections inherent in the real estate market. While previous panel-based unit root tests have been joint tests of a unit root for all members of a panel and have not been capable of determining the mix of $\mathrm{I}(0)$ and I(1) series in a panel setting, the SPSM, proposed by Chortareas and Kapetanios (2009), classifies a whole panel into a group of stationary members and a group of non-stationary members. This clearly identifies how many and which series in the panel are stationary processes. The combined use of the panel KSS test with a Fourier function and the SPSM lead us to conclude that stationarity is not influenced across provinces and distinct sizes (hence income levels).

The paper contributes to the literature by adopting the SPSM to test the stationarity of house prices for a sample from 9 provinces over the period from 1978Q1 to 2012.Q4. The combined use of the Panel KSS test with a Fourier function and the SPSM allows us to obtain clear conclusions about the stationarity of house prices in our study.

\section{References}

Bai, J. and Ng. S. 2004. "A Panic Attack on Unit Roots and Cointegration." Econometrica, 72:1127-1177.

Balcilar, M., Beyene, A. D., Gupta, R. and Seleteng, M. 2013. Ripple effects in the South African house prices. Urban Studies, 50(5):876-894.

Balcilar, M., Gupta, R. and. Shah, Z. 2011. An In-Sample and Out-of-Sample Empirical Investigation of the Nonlinearity in House Prices of South Africa. Economic Modelling, 28(3):891-899. 
Becker, R., Enders, W. and Lee, J. 2004. A general test for time dependence in parameters, Journal of Applied Econometrics, 19:899-906.

Becker, R., Enders, W. and Lee, J. 2006. A stationairy test in the presence of an unknown number of smooth breaks, Journal of Time Series Analysis, 27(3):381-409.

Breuer, J.B., McNown, R., and Wallace, M.S. 2001. Misleading inferences from panel unit root tests with an illustration from purchasing power parity, Review of International Economics, 9:482-493.

Bierens, H.J. 1997. Testing the unit root with drift hypothesis against nonlinear trend stationarity, with an application to the U.S. price level and interest rate, Journal of Econometrics, 81:29-64.

Burger, P. and van Rensburg, L.J. 2008. Metropolitan house prices in South Africa: Do they converge? South African Journal of Economics, 76(2):291-297.

Canarella, G., Miller, S. M. and Pollard, S. K. 2012. Unit Roots and Structural Change: An Application to US House-Price Indices. Urban Studies, 49(3):757-776.

Carrion-i-Silvestre, J.L., del Barrio-Castro, T. and Lopez-Bazo, E. 2005. Breaking the panels: An application to the GDP per capita. Econometrics Journal, Royal Economic Society, 8(2):159-175.

Chang T., Liu, W-C., Aye, G.C. and Gupta, R. forthcoming. .Are there housing bubbles in South Africa? evidence from SPSM-based panel KSS test with a fourier function."Global Business and Economics Review.

Chang Y. 2002. Nonlinear IV unit root tests in panels with cross-sectional dependency. Journal of Econometrics, 110: 261-292.

Choi, I. 2002. "Combination Unit Root Tests for Cross-Sectionally Correlated Panels." in The Econometric Theory and Practice: Frontiers of Analysis and Applied Research, Essays in Honor of Peter C. B. Phillips. D. Corbae, S.N. Durlauf and B.E. Hansen (eds.). Cambridge University Press, Cambridge, UK, 311-333.

Chortareas, G. and Kapetanios, G. 2009. "Getting PPP right: identifying mean-reverting real exchange rates in panels." Journal of Banking and Finance, 33:390-404.

Cook, S. and Vougas, D. 2009. Unit Root Testing against an ST-MTAR Alternative: Finite-Sample Properties and an Application to the UK Housing Market. Applied Economics, 41:1397-1404.

Das, S., Gupta, R. and Kaya, P.A. 2010. Convergence of metropolitan house prices in South Africa: A re-examination using efficient unit root tests. Applied Econometrics and International Development, 10(1):173-188.

Diebold, F.X. and Kilian, L. 2000. Unit root tests are useful for selecting forecasting models. Journal of Business and Economic Statistics, 18:265-273.

Dickey, D.A. and Fuller, W.A. 1981. Likelihood ratio statistics for autoregressive time series with a unit root. Econometrica, 49:1057-1072.

Elliott, G., Rothenberg, T. J. and Stock, J. H. 1996. Efficient Tests for an Autoregressive Unit Root. Econometrica, 64:813-836.

Enders, W. and Lee, J. 2004. Testing for a unit root with a nonlinear Fourier function, Working Paper, Department of Economics, Finance \& Legal Studies, University of Alabama, Tuscaloosa, AL, USA.

Enders, W. and Lee, J. 2009. The flexible Fourier form and testing for unit roots: An example of the term structure of interest rates, Working Paper, Department of Economics, Finance \& Legal Studies, University of Alabama, Tuscaloosa, AL, USA. 
Enders, W. and Lee, J. 2012. A Unit Root Test Using a Fourier Series to Approximate Smooth Breaks. Oxford Bulletin of Economics and Statistics, 74(4):574-599.

Gallant, R. 1981. On the basis in flexible functional form and an essentially unbiased form: The flexible Fourier form, Journal of Econometrics, 15:211-353.

Gil-Alana, L.A., Aye, G.C. and Gupta, R. forthcoming. Testing for Persistence with Breaks and Outliers in South African House Prices. Journal of Real Estate Literature.

Hadri, K. 2000. Testing for Stationarity in Heterogeneous Panel Data. Econometrics Journal, 3: 148-161.

Gupta, R. and Hartley, F. forthcoming. The Role of Asset Prices in Forecasting Inflation and Output in South Africa. Journal of Emerging Market Finance.

Im, K. S., Pesaran, M.H. and Shin, Y. 2003. "Testing for Unit Roots in Heterogeneous Panels." Journal of Econometrics, 115:53-74.

Kapetanios, G., Shin, Y., and Snell, A. 2003. Testing for Cointegration in Nonlinear Smooth Transition Error Correction Models. Econometric Theory, 22:279-303.

Kim, S.W. and Bhatttacharya, R. 2009. Regional housing prices in the USA: An empirical investigation of the nonlinearity. Journal of Real Estate Finance and Economics, 38:443-460.

Kwiatkowski, D., Phillips, P., Schmidt, P. and Shin, J. 1992. Testing the Null Hypothesis of Stationarity against the Alternative of a Unit Root. Journal of Econometrics, 54:159-178.

Lee, J. and Strazicich, M. C. 2003. Minimum LM Unit Root Test with Two Structural Breaks. Review of Economics and Statistics, 85:1082-1089.

Levin, A., Lin, C.F. and Chia-Shang Chu. 2002. "Unit Root in Panel Data: Asymptotic and Finite-Sample Properties." Journal of Econometrics, 108:1-24.

Leybourne, S., Newbold, P. and Vougas, D. 1998. Unit Roots and Smooth Transitions. Journal of Time Series Analysis, 19:83-98.

Lumsdaine, R. and Papell, D. 1997. Multiple Trend Breaks and the Unit Root Hypothesis. Review of Economics and Statistics, 79:212-218.

Maddala, G. and Kim, I.-M. 1998. Unit roots, cointegration and structural change, Cambridge University Press, UK.

Maddala, G.S. and Wu, S. 1999. "A Comparative Study of Unit Root Tests with Panel Data and a New Simple Test." Oxford Bulletin of Economics and Statistics, 61: 631-652.

Meen, G. 1999. Regional House Prices and the Ripple Effect: A New Interpretation. Housing Studies, 14:733-753.

Meen, G. 2002. The Time-Series Properties of House Prices: A Transatlantic Divide? Journal of Housing Economics, 11:1-23. 33

Moon, H. R. and Perron, B. 2004. "Testing for a Unit Root in Panels with Dynamic Factors.” Journal of Econometrics, 122:81-126.

Muellbauer, J. and Murphy, A. 1997. Booms and busts in the UK housing market. The Economic Journal, 445:1701-1727.

Muñoz, S. 2003. Real Effects of Regional House Prices: Dynamic Panel Estimation with Heterogeneity. mimeo, International Monetary Fund and London School of Economics.

Nelson, C. R., and Plosser, C. I. 1982. Trends and random walks in macroeconomic time series: Some evidence and implications. Journal of Monetary Economics, 10:139-162. 
Newey, W., and West, K. 1994. Automatic Lag Selection in Covariance Matrix Estimation. Review of Economic Studies, 61: 631-653.

Pascalau, R. 2010. Unit root tests with smooth breaks: an application to the Nelson-Plosser data set, Applied Economics Letters, 17:565-570.

Perron, P. 1989. The great crash, the oil price shock and the unit root hypothesis, Econometrica, 57: 1361-401.

Pesaran, M.H. 2004. “General Diagnostic Tests for Cross Section Dependence in Panels." IZA Discussion Papers 1240, Institute for the Study of Labor (IZA).

Pesaran, M.H. 2007. “A Simple Unit Root Test in the Presence of Cross-Section Dependence.” Journal of Applied Econometrics, 22:265-312.

Peterson, W., Holly, S., and Gaudoin, P. 2002. Further Work on an Economic Model of the Demand for Social Housing. Report to the Department of the Environment, Transport and the Regions.

Phillips, P. C. B., and Perron, P. 1988. Testing for a Unit Root in Time Series Regression. Biometrika, 75:335-346.

Seslen, N. 2004. Housing price dynamics and household mobility decisions. Seminar Paper,USC Lusk/FBE Real Estate Seminar, September.

Simo-Kengne, B.D., Bittencourt, M. and Gupta, R. 2012. House prices and economic growth in South Africa: Evidence from provincial-level data. Journal of Real Estate Literature, 20(1):97-117.

Simo-Kengne, B.D., Bittencourt, M. and Gupta, R. 2013. The impact of house prices on consumption in South Africa: Evidence from provincial-level panel VARs. Housing Studies, 28(8): 1133-1154.

Simo-Kengne, B.D.,Gupta, R. and Aye, G.C. forthcoming a. Macro shocks and house prices in South Africa. Journal of Real Estate Portfolio Management.

Simo-Kengne, B.D.,Gupta, R. and Aye, G.C. forthcoming b. House prices and balance of trade dynamics in South Africa: evidence from an agnostic identification Procedure. Journal of Housing Research.

Taylor, M.P. and Sarno, L. 1998. The behavior of real exchanges during the post-Bretton Woods period, Journal of International Economics, 46:281-312.

Ucar, N. and Omay, T. 2009. Testing for unit root in nonlinear heterogeneous panels, Economics Letters, 104:5-8.

Wu, J.L. and Lee, H.Y. 2009. A revisit to the non-linear mean reversion of real exchange rates: evidence from a series-specific non-linear panel unit-root test, Journal of Macroeconomics, 31:591-601. 